\title{
Resultados del tratamiento del microcarcinoma papilar de tiroides adaptado al riesgo de recurrencia
}

\author{
Results of treatment of papillary thyroid microcarcinoma adapted to risk of recurrence
}

\author{
José Ruiz-Pardo ${ }^{1 *}$, Antonio Ríos-Zambudio, José M. Rodríguez-González¹, Miriam Paredes-Quiles', \\ Víctor Soriano-Giménez ${ }^{1}$, M. Isabel Oviedo-Ramírez², Antonio M. Hernández-Martínez y y \\ Pascual Parrilla-Paricio ${ }^{1}$ \\ ${ }^{1}$ Servicio de Cirugía General y del Aparato Digestivo; ${ }^{2}$ Servicio de Anatomía Patológica; ${ }^{3}$ Servicio de Endocrinología y Nutrición. Hospital Clínico \\ Universitario Virgen de la Arrixaca, Murcia, España
}

\begin{abstract}
Resumen
Antecedentes: La incidencia del microcarcinoma papilar de tiroides (MCPT) está aumentado. Objetivo: Analizar el pronóstico a largo plazo del MCPT. Método: Población a estudio: pacientes con diagnóstico histopatológico de MCPT (tamaño $\leq 1 \mathrm{~cm}$ ) tratados según el riesgo de recurrencia de la Sociedad Latinoamericana de Tiroides. Criterios de inclusión: seguimiento mínimo de 2 años, disponibilidad de las muestras histopatológicas y cumplimiento del tratamiento. Criterios de exclusión: cirugía tiroidea previa, otras patologías malignas sincrónicas o localización ectópica del MCPT. Variables a estudio: persistencias, recidivas y mortalidad. Resultados: Según el riesgo de recurrencia, el $65.2 \%(n=105)$ tuvo muy bajo riesgo, el 17.4\% $(n=28)$ bajo riesgo y el $17,4 \%(n=28)$ alto riesgo. En los pacientes de alto riesgo se realizó tiroidectomía total en todos los casos, linfadenectomía cervical en el $57,1 \%(n=16)$ y terapia metabólica con $l^{131}$ en todos los casos. Durante un seguimiento medio de 119,8 \pm 65 meses hubo un $0,6 \%(n=1)$ de recurrencias. No se evidenciaron factores de riesgo asociados a recidiva de la enfermedad. Ningún paciente falleció debido al MCPT. Conclusiones: EI MCPT tratado en función del riesgo de recurrencia tiene un buen pronóstico a largo plazo, sin persistencias, con una baja cifra de recurrencias y ausencia de mortalidad debida a la enfermedad.
\end{abstract}

Palabras clave: Cáncer de tiroides. Carcinoma papilar de tiroides. Microcarcinoma papilar de tiroides. Pronóstico. Tratamiento.

\begin{abstract}
Background: The incidence of papillary thyroid microcarcinoma (PTMC) is increasing. Objective: To analyze the long-term prognosis of PTMC. Method: Study population: patients with a histopathological diagnosis of PTMC (size $\leq 1 \mathrm{~cm})$ treated according to the risk of recurrence of the Latin American Thyroid Society. Inclusion criteria: minimum follow-up of 2 years, availability of histopathological samples, and treatment compliance. Exclusion criteria: previous thyroid surgery, other synchronous malignancies or ectopic location of the PTMC. Study variables: persistences, recurrences and mortality. Results: Based on the risk of recurrence, PTMC has very low risk in $65.2 \%(n=105)$, low risk in $17.4 \%(n=28)$ and high risk in $17.4 \%$ $(n=28)$. In high risk patients, total thyroidectomy was performed in all cases, cervical lymphadenectomy in $57,1 \%(n=16)$ and metabolic therapy with $I^{131}$ in all cases. During a mean follow-up of 119,8 \pm 65 months, $0.6 \%(n=1)$ of recurrences took place. Risk factors associated to recurrence were not identified. No patient died due to MCPT. Conclusions: PTMC treated
\end{abstract}

\footnotetext{
Correspondencia:

*José Ruiz Pardo

Ctra. Madrid-Cartagena, s/n

Fecha de recepción: 04-09-2019

Cir Cir. 2020;88(5):576-583

C.P. 30120, El Palmar, Murcia, España

Fecha de aceptación: 24-05-2020

Contents available at PubMed

E-mail: josrp@ hotmail.es

DOI: $10.24875 / C I R U .20001560$

www.cirugiaycirujanos.com

0009-7411/@ 2020 Academia Mexicana de Cirugía. Publicado por Permanyer. Este es un artículo open access bajo la licencia CC BY-NC-ND (http://creativecommons.org/licenses/by-nc-nd/4.0/).
} 
based on its risk of recurrence has a good long-term prognosis, without persistences, with a low number of recurrences and absence of disease-associated mortality.

Key words: Thyroid cancer. Papillary thyroid carcinoma. Papillary thyroid microcarcinoma. Prognosis. Treatment.

\section{Introducción}

El microcarcinoma papilar de tiroides (MCPT) es aquel carcinoma papilar que mide $\leq 1 \mathrm{~cm}^{1}$. Su incidencia está aumentando considerablemente en los últimos años ${ }^{2}$, no solo por un aumento real, sino también por un mejor estudio clínico preoperatorio ${ }^{3-5}$ y un diagnóstico histopatológico más preciso en pacientes que se intervienen por patología tiroidea presumiblemente benigna ${ }^{6-9}$.

Debido al incremento de su incidencia, es importante conocer su pronóstico para poder realizar un tratamiento adecuado, sin ser excesivo ni escaso. Actualmente, el tratamiento tiende a ser cada vez menos agresivo y más conservador, minimizando el riesgo de complicaciones posquirúrgicas e intentando disminuir el número de persistencias, recurrencias y mortalidad relacionada con la enfermedad. Sin embargo, no hay un consenso acerca de cuál es el mejor tratamiento $0^{10-15}$, ya que existen opciones desde muy conservadoras, sobre todo en centros asiáticos donde solo se realiza un seguimiento activo ${ }^{10}$, hasta completas con cirugía (lobectomía o tiroidectomía total, linfadenectomía) y radioyodo $\left(I^{131}\right)^{11-15}$.

Ante esta variabilidad terapéutica, lo más razonable sigue siendo intentar identificar aquellos MCPT que pueden presentar un mal pronóstico y en los que se debería realizar un tratamiento más agresivo, de tal manera que no se haga un sobretratamiento de los casos más indolentes ni se dejen de tratar adecuadamente los tumores más agresivos. En este sentido, cada vez son más los centros que aconsejan un tratamiento individualizado adaptado al riesgo de recurrencia del tumor ${ }^{16}$.

El objetivo de este estudio es analizar el tratamiento del MCPT según el riesgo de recurrencia y su pronóstico a largo plazo.

\section{Método}

\section{Población a estudio}

La población la constituyen los pacientes con diagnóstico histopatológico de MCPT (carcinoma papilar $\leq 1 \mathrm{~cm}$ ) en el periodo comprendido entre $1995 \mathrm{y}$ 2015, tratados en función del riesgo de recurrencia en una unidad de cirugía endocrina. Se realiza un análisis retrospectivo de la base de datos recolectados en la unidad de cirugía endocrina.

Dentro de la población a estudio se seleccionan aquellos pacientes que cumplen los siguientes criterios de inclusión: seguimiento mínimo de 2 años, disponibilidad de las muestras histopatológicas en el archivo del servicio de anatomía patológica y cumplimiento del tratamiento acordado por el comité de cirugía endocrina en función del riesgo de recurrencia.

Dentro de la población seleccionada se excluyen los pacientes con cirugía tiroidea previa a la cirugía del MCPT, presencia de otras patologías tiroideas o extratiroideas malignas sincrónicas, localización ectópica del MCPT (sublingual, quiste tirogloso, struma ovarii, etc.) o historia clínica incompleta.

\section{Valoración del riesgo de recurrencia del MCPT}

El riesgo de recurrencia se evalúa según la clasificación de la Sociedad Latinoamericana de Tiroides $(\mathrm{SLAT})^{16}$ :

- Muy bajo riesgo de recurrencia: tumor $\leq 1 \mathrm{~cm}$ unifocal, sin extensión más allá de la cápsula tiroidea y sin metástasis ganglionares ni a distancia.

- Bajo riesgo de recurrencia: tumor $\leq 1 \mathrm{~cm}$ multifocal, sin extensión más allá de la cápsula tiroidea ni metástasis ganglionares ni a distancia.

- Alto riesgo de recurrencia: tumor con metástasis ganglionares regionales, enfermedad persistente, invasión extracapsular a los tejidos y estructuras locorregionales, invasión vascular, metástasis a distancia (M1), histología agresiva del tumor (por ejemplo, CPT de células altas, de células columnares, tipo insular, etc...), o captación de ${ }^{131}$ fuera de la celda tiroidea en la primera gammagrafía postratamiento.

\section{Tratamiento adaptado al riesgo de recurrencia}

1) Tratamiento quirúrgico

El tratamiento quirúrgico está condicionado por el diagnóstico del MCPT. Si el diagnóstico es clínico preoperatorio, la técnica quirúrgica se decide en 
dependencia de las características ecográficas del MCPT y de la presencia de adenopatías cervicales sospechosas o positivas para malignidad. Si el diagnóstico es incidental en la pieza de tiroidectomía en pacientes que son intervenidos por patología tiroidea presumiblemente benigna, se decide si la técnica quirúrgica inicial es suficiente o debe completarse de acuerdo con los hallazgos histopatológicos.

2) Valoración del tratamiento con ${ }^{131}$

La ablación de restos tiroideos con $\mathrm{I}^{131}$ se realiza en pacientes sometidos a tiroidectomía total, en función del riesgo de recurrencia, siempre y cuando no existan contraindicaciones para su administración (alergia, embarazo y lactancia materna):

- En los pacientes con muy bajo riesgo de recurrencia no se administra $I^{131}$.

- En los pacientes con bajo riesgo de recurrencia se valora cada caso individualmente, analizando los riesgos y los beneficios de la administración de ${ }^{131}$. En pacientes en edad fértil que quieran tener descendencia se valora su administración.

- En los pacientes con alto riesgo de recurrencia siempre se administra $I^{131}$.

3) Valoración de la terapia de supresión de tirotropina La terapia de supresión de tirotropina (TSH, thyroid-stimulating hormone) se realiza con levotiroxina (LT4) en función del riesgo de recurrencia, siempre y cuando no esté contraindicada (cardiopatía isquémica, fibrilación auricular y osteoporosis):

- En los pacientes con muy bajo riesgo de recurrencia no se realiza la supresión de TSH.

- En los pacientes con bajo riesgo de recurrencia y sin evidencia de recidiva locorregional o metástasis a distancia se realiza la supresión de TSH manteniendo sus valores entre 0.4 y $1.0 \mathrm{mUI} / \mathrm{l}$.

- En los pacientes con alto riesgo de recurrencia se realiza la supresión de TSH manteniendo sus valores $\leq 0.1 \mathrm{mUl} / \mathrm{l}$.

\section{Criterios de curación y detección de persistencias y recidivas}

1) Criterios de curación

Se considera que se cumplen criterios de curación tras el tratamiento cuando a los 6 meses:

- En pacientes sometidos a lobectomía o tiroidectomía total sin ablación de restos tiroideos con li31:

- No existe evidencia clínica de enfermedad activa.

- La ecografía cervical es normal.
- En pacientes sometidos a tiroidectomía total con ablación de restos tiroideos con I $^{131}$ :

- No existe evidencia clínica de enfermedad activa.

- Existen uno o dos rastreos con $\mathrm{I}^{131}$ negativos.

- La tiroglobulina es $<2 \mathrm{ng} / \mathrm{ml}$ (tras la retirada de LT4 o tras estimulación con TSH recombinante humana [rhTSH]) y los anticuerpos antitiroglobulina son negativos.

- La ecografía cervical es normal.

2) Persistencia de la enfermedad

Se define persistencia como la presencia de evidencia clínica, bioquímica o radiológica de enfermedad (ecografía, tomografía computarizada [TC], gammagrafía de cuerpo completo con $\mathrm{I}^{131} \mathrm{o}$ tomografía por emisión de positrones [PET]) en los primeros 6 meses después de la cirugía. En los pacientes sometidos a lobectomía, en los que se completa la tiroidectomía en los primeros 6 meses y se evidencia un MCPT, no se considera como una persistencia de la enfermedad, sino como un MCPT multicéntrico tratado con una tiroidectomía total.

3) Recidiva de la enfermedad

Se define recidiva como la presencia de evidencia clínica, bioquímica o radiológica de enfermedad (ecografía, TC, gammagrafía de cuerpo completo con ${ }^{131}$ o PET) a partir de los 6 meses siguientes a la cirugía. Se diferencian tres tipos:

- Recidiva bioquímica: se define como cualquier medición repetida de tiroglobulina $>2 \mathrm{ng} / \mathrm{ml}$ (estimulada con rhTSH o tras la retirada de la terapia de supresión de la TSH), considerándose únicamente en pacientes a quienes se realiza una tiroidectomía total y ablación de restos tiroideos con $\mathrm{I}^{131}$.

- Recidiva locorregional: se evidencia por pruebas de imagen (ecografía, gammagrafía, TC o PET) y tiene que ser confirmada en el análisis histopatológico después de una punción-aspiración con aguja fina (PAAF), biopsia o cirugía.

- Recidiva a distancia: debe ser confirmada mediante análisis histológico después de realizar una PAAF, biopsia o cirugía, o mediante gammagrafía de ${ }^{131}$ o PET de cuerpo entero.

\section{Variables a estudio}

Se analizaron las siguientes variables:

- Socio-personales: edad y sexo.

- Terapéuticas: técnica quirúrgica (tiroidectomía y linfadenectomía cervical), ablación de restos tiroideos con $\mathrm{I}^{131}$ y terapia de supresión de TSH. 
- Histopatológicas: tamaño, focalidad, lateralidad, variantes histopatológicas, invasión extracapsular, márgenes de resección y adenopatías metastásicas.

- Seguimiento: riesgo de recurrencia, persistencias, recidivas, supervivencia global y mortalidad relacionada con la enfermedad.

\section{Método estadístico}

Los datos fueron recogidos en una base de datos. Para variables categóricas, los datos se expresan mediante frecuencias y porcentajes, y son comparados mediante el test de ji al cuadrado de Pearson o el test exacto de Fisher cuando es apropiado. Para las variables cuantitativas continuas, los datos se expresan como media \pm desviación estándar. Se utilizan el método de Kaplan-Meier para analizar la supervivencia global y el test log rank para su comparación entre grupos. Un valor de $p<0.05$ se considera como estadísticamente significativo.

\section{Resultados}

\section{Descripción de la serie}

Cumplieron los criterios de selección 161 pacientes. La edad media fue de $46.2 \pm 13$ años (16-81) y el $80.7 \%(n=130)$ eran mujeres. El $54 \%(n=87)$ de los casos fueron incidentales, es decir, diagnosticados en el análisis histopatológico de los pacientes intervenidos por patología tiroidea presumiblemente benigna. En la tabla 1 se detallan las características histopatológicas del MCPT.

Según la clasificación de la SLAT, el 65.2\% $(n=105)$ de los tumores fueron de muy bajo riesgo de recurrencia, el $17.4 \%(n=28)$ de bajo riesgo de recurrencia y el $17.4 \%(n=28)$ de alto riesgo de recurrencia.

El número de tiroidectomías, lobectomías y linfadenectomías cervicales realizadas en función del riesgo de recurrencia se detalla en la figura 1.

En cuanto a la linfadenectomía cervical, se realizó con más frecuencia en los pacientes de alto riesgo que en los de bajo y muy bajo riesgo (57.1 vs. 7.1 vs. $3,8 \%$, respectivamente; $p<0.001$ ).

La administración $I^{131}$ según el riesgo de recurrencia se expone en la figura 2 . Se administró $I^{131}$ a más pacientes con alto riesgo de recurrencia que a los de bajo y muy bajo riesgo de recurrencia (100 vs. 82.1 vs. $0 \%$, respectivamente; $p<0.001$ ).
Tabla 1. Características histopatológicas del microcarcinoma papilar de tiroides

\begin{tabular}{lc}
\hline Variables histopatológicas & $\mathbf{n}(\%)$ \\
\hline Tamaño $(\mathrm{mm})$ & $5.3 \pm 3(0.7-10)$ \\
Focalidad & \\
$\quad$ Unifocal & $117(72.7 \%)$ \\
Multifocal & $44(27.3 \%)$ \\
Lateralidad & \\
$\quad$ Unilateral & $136(84.5 \%)$ \\
Bilateral & $25(15.5 \%)$ \\
Subtipos histológicos & \\
Papilar clásico & $82(51 \%)$ \\
Folicular & $76(47.2 \%)$ \\
Oncocítico & $1(0.6 \%)$ \\
Células altas & $1(0.6 \%)$ \\
Sólido & $1(0.6 \%)$ \\
Invasión extracapsular & \\
No & $143(88.8 \%)$ \\
Sí & $18(11.2 \%)$ \\
Márgenes de resección & \\
No afectados & $154(95.7 \%)$ \\
Afectados & $7(4.3 \%)$ \\
Adenopatías metastásicas & \\
No & $146(90.7 \%)$ \\
Sí & $15(9.3 \%)$ \\
\hline
\end{tabular}

Todos los pacientes con alto riesgo $(n=28)$ y bajo riesgo $(n=28)$ de recurrencia recibieron terapia de supresión de TSH con LT4. Los pacientes con muy bajo riesgo de recurrencia no recibieron terapia de supresión de TSH.

\section{Pronóstico del MCPT}

Durante un seguimiento medio de $119.8 \pm 65$ meses (24-271) no hubo persistencias de la enfermedad, pero sí ocurrió una recurrencia $(0.6 \%)$, que se produjo a los 96 meses de seguimiento en una mujer de 44 años con diagnóstico clínico de MCPT, en la que se realizó una tiroidectomía total más vaciamiento central homolateral. En el análisis histopatológico se evidenció un MCPT de variante clásica, unifocal, de $8 \mathrm{~mm}$ de diámetro máximo, con invasión extracapsular mínima y con 3/11 adenopatías positivas para malignidad (Fig. 3).

En los pacientes sometidos a lobectomía a quienes no se completó la tiroidectomía $(n=11)$ no se evidenciaron recidivas en el remanente tiroideo durante un seguimiento medio de $116.8 \pm 58.5$ meses.

No se objetivaron diferencias significativas respecto a la supervivencia global según el riesgo de recurrencia 


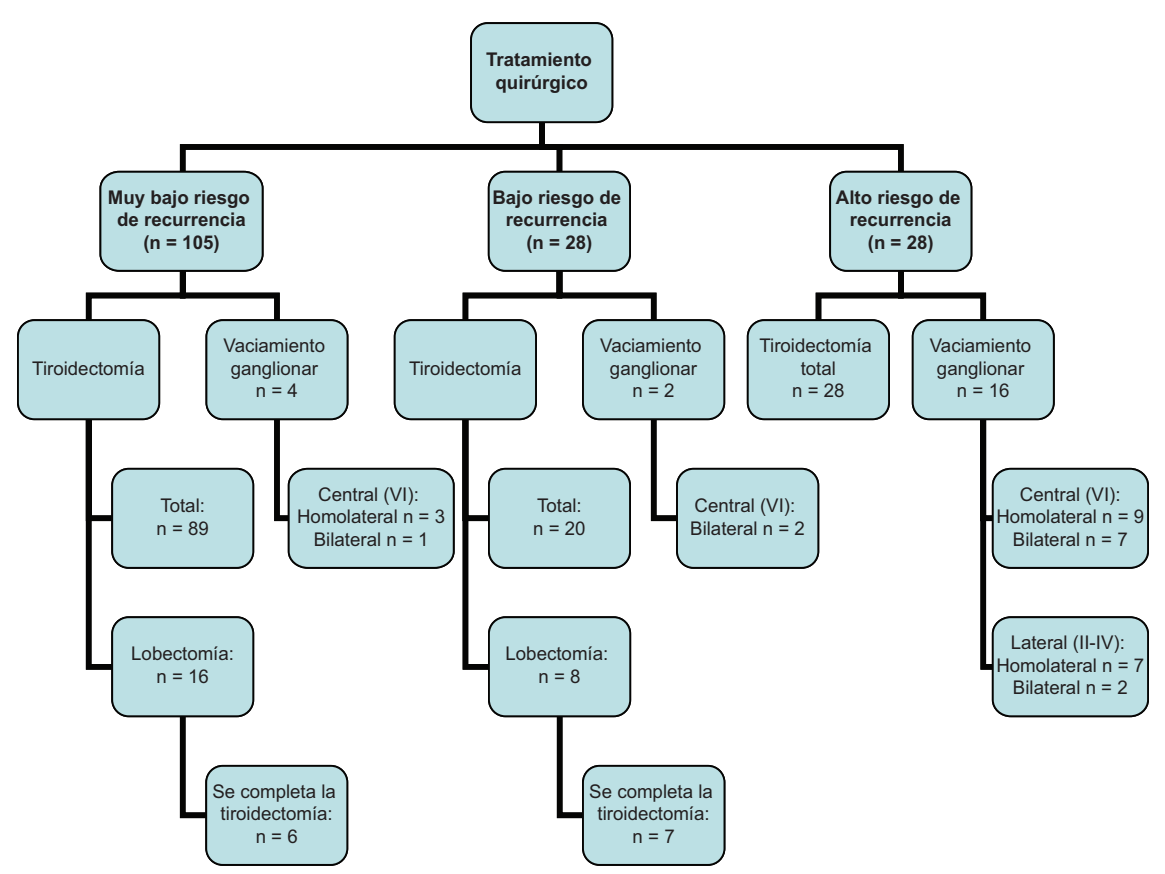

Figura 1. Técnica quirúrgica en función del riesgo de recurrencia.

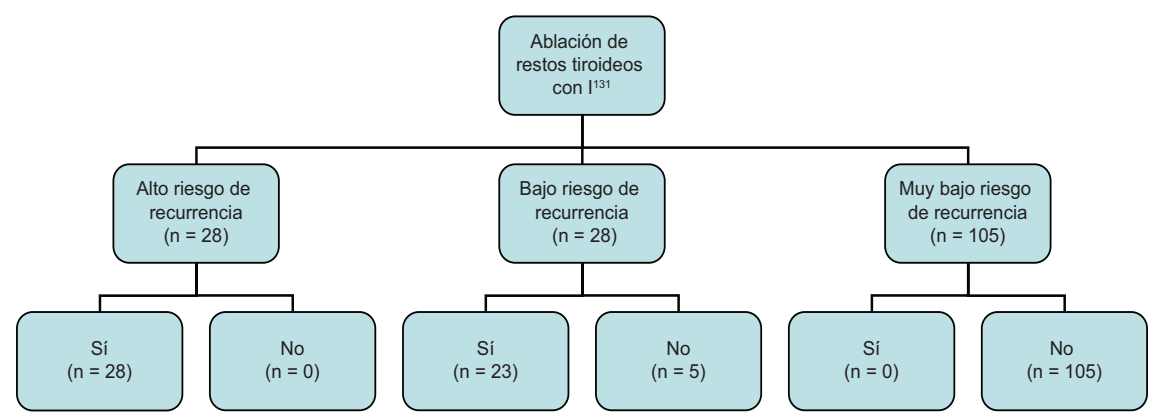

Figura 2. Ablación de restos tiroideos con $l^{131}$ en función del riesgo de recurrencia.

(muy bajo riesgo $123.3 \pm 6.2$ meses vs. bajo riesgo $121.1 \pm 13.1$ vs. alto riesgo $120.4 \pm 13.4$ meses; $\mathrm{p}=0.996)$ (Fig. 4).

Aunque no hubo mortalidad relacionada con la enfermedad, el $3.1 \%(n=5)$ de los pacientes fallecieron durante el seguimiento por causas ajenas a la enfermedad.

Al analizar los factores asociados con la presencia de recidiva, no se encontraron diferencias significativas en ninguna de las variables analizadas (Tabla 2).

\section{Discusión}

El aumento de la incidencia del MCPT en los últimos años ${ }^{2}$, junto con su pronóstico generalmente favorable, han llevado a establecer protocolos adecuados de tratamiento, de tal manera que el tratamiento inicial no sea muy agresivo ni tampoco insuficiente, minimizando las complicaciones posquirúrgicas, las persistencias, las recidivas y la mortalidad relacionada con la enfermedad.

Al tratarse de un estudio de cohorte retrospectiva hay que aclarar determinados aspectos que podrían dar lugar a varios sesgos. En primer lugar, los pacientes analizados en el estudio son de un lugar específico, en concreto de la Región de Murcia (España). También hay que mencionar que el periodo en que los pacientes han sido seleccionados ha sido largo, desde 1995 hasta 2015, por lo que los métodos diagnósticos y terapéuticos podrían haber variado. Los métodos diagnósticos apenas han cambiado desde 1995, ya que por entonces ya se realizaba la ecografía cervical, 

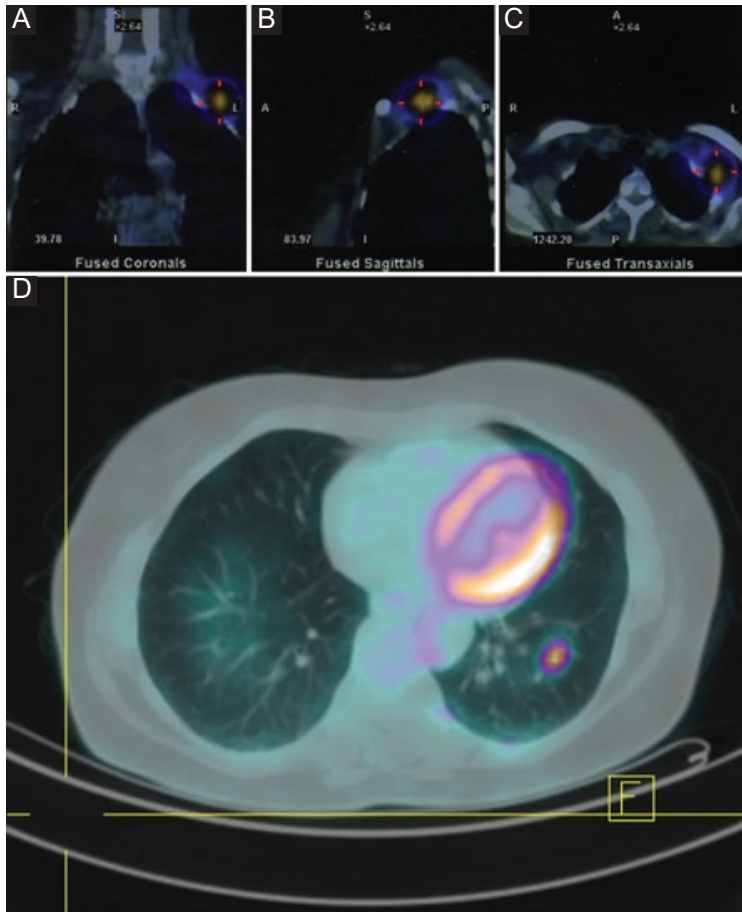

Figura 3. A-C: cortes coronal, sagital y axial de la SPECT, en los que se evidencia una captación más intensa a nivel infraclavicular izquierdo, compatible con recidiva en esa localización. D: corte axial de la PET en la que se evidencia un incremento patológico del metabolismo glucídico (SUVmax de 6,1) en una lesión metastásica de $1,2 \mathrm{~cm}$ en el lóbulo pulmonar inferior izquierdo. SPECT: single photon emission computed tomography.

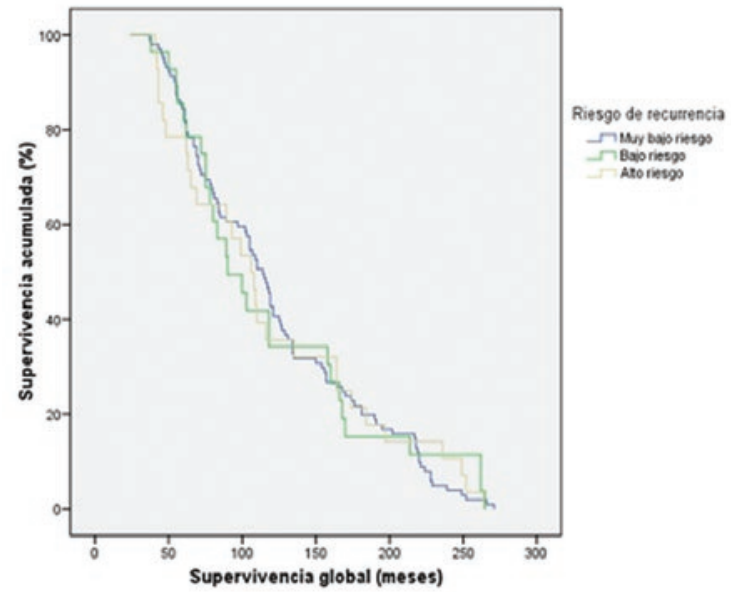

Figura 4. Supervivencia global según el riesgo de recurrencia.

y si el nódulo tiroideo o la adenopatía cervical eran sospechosos de malignidad se realizaba biopsia mediante PAAF, y dependiendo de la sospecha o la confirmación de malignidad en el análisis citológico se realizaba la cirugía. También existen un $54 \%$ de casos incidentales, es decir, aquellos en los que el
Tabla 2. Factores asociados a recidiva del microcarcinoma papilar de tiroides

\begin{tabular}{|c|c|c|c|}
\hline $\begin{array}{l}\text { Variables } \\
\text { histopatológicas }\end{array}$ & $\begin{array}{l}\text { Sin recidiva } \\
(\mathrm{n}=160)\end{array}$ & $\begin{array}{c}\text { Recidiva } \\
(\mathrm{n}=1)\end{array}$ & $p$ \\
\hline \multicolumn{4}{|l|}{ Edad } \\
\hline$<45$ años & $44.4 \%(n=71)$ & $100 \%(n=1)$ & 0.447 \\
\hline$\geq 45$ años & $55.6 \%(n=89)$ & $0 \%$ & \\
\hline \multicolumn{4}{|l|}{ Sexo } \\
\hline Mujeres & $80.6 \%(n=129)$ & $100 \%(n=1)$ & 1.000 \\
\hline Hombres & $19.4 \%(n=31)$ & $0 \%$ & \\
\hline \multicolumn{4}{|c|}{ Tamaño tumoral $\geq 8 \mathrm{~mm}$} \\
\hline No & $75.6 \%(n=121)$ & $0 \%$ & 0.248 \\
\hline Sí & $24.4 \%(n=39)$ & $100 \%(n=1)$ & \\
\hline \multicolumn{4}{|l|}{ Focalidad } \\
\hline Unifocal & $72.5 \%(n=116)$ & $100 \%(n=1)$ & 1.000 \\
\hline Multifocal & $27.5 \%(n=44)$ & $0 \%$ & \\
\hline \multicolumn{4}{|l|}{ Lateralidad } \\
\hline Unilateral & $84.4 \%(n=135)$ & $100 \%(n=1)$ & 1.000 \\
\hline Bilateral & $15.6 \%(n=25)$ & $0 \%$ & \\
\hline \multicolumn{4}{|c|}{ Invasión extracapsular } \\
\hline No & $88.7 \%(n=143)$ & $0 \%$ & 0.112 \\
\hline Sí & $11.3 \%(n=17)$ & $100 \%(n=1)$ & \\
\hline \multicolumn{4}{|c|}{ Márgenes de resección } \\
\hline No afectados & $95.6 \%(n=153)$ & $100 \%(n=1)$ & 1.000 \\
\hline Afectados & $4.4 \%(n=7)$ & $0 \%$ & \\
\hline \multicolumn{4}{|c|}{ Metástasis ganglionares } \\
\hline No & $91.2 \%(n=146)$ & $0 \%$ & 0.093 \\
\hline Sí & $8.8 \%(n=14)$ & $100 \%(n=1)$ & \\
\hline
\end{tabular}

diagnóstico de MCPT se realizó en el análisis histopatológico de muestras de pacientes intervenidos por patología tiroidea presumiblemente benigna. Solo habría que añadir que con los años se ha introducido la ecografía cervical de alta resolución, que permite identificar nódulos de menor tamaño y dirigir mejor su PAAF. Respecto al tratamiento del MCPT, tampoco ha habido grandes cambios en nuestro hospital durante el tiempo que ha durado el estudio. En los casos de MCPT con diagnóstico clínico se realiza una tiroidectomía total. Además, se lleva a cabo vaciamiento cervical central o lateral, o ambos, dependiendo de la presencia de adenopatías metastásicas (diagnóstico preoperatorio por PAAF de una adenopatía metastásica o diagnóstico intraoperatorio mediante congelación cuando existe alta sospecha). Siempre que se realiza un vaciamiento laterocervical se hace también un vaciamiento central homolateral. En los casos en que el diagnóstico es incidental se decide si la técnica quirúrgica inicial (lobectomía o tiroidectomía total) es suficiente 0 debe completarse en función de los 
hallazgos histopatológicos. A los pacientes con MCPT con muy bajo riesgo de recurrencia no se les administran tratamientos complementarios a la cirugía; a los de bajo riesgo de recurrencia se les administra I $^{131}$ valorando los riesgos y los beneficios individualmente, y se realiza terapia de supresión de TSH; y a los de alto riesgo de recurrencia siempre se les administra ${ }^{131}$ y se realiza terapia de supresión de TSH. Por otro lado, hay que aclarar que todos los pacientes diagnosticados de MCPT fueron tratados y posteriormente seguidos en la consulta hasta el final del estudio, salvo cinco que fallecieron durante el seguimiento por causas ajenas a la enfermedad. Por último, para evitar sesgos de información, los pacientes con una historia clínica incompleta y aquellos en los que faltaban datos de las variables consideradas fueron excluidos del estudio, lo que refleja la adecuada validez de los datos.

El hecho de que el pronóstico del MCPT sea generalmente indolente no justifica un tratamiento sistemático poco agresivo (sin linfadenectomía ni ablación de restos tiroideos con $\mathrm{I}^{131}$ ). En la serie presentada, solo el $17.4 \%$ de los MCPT fueron de alto riesgo de recurrencia, por lo que los resultados aportados en este estudio avalan un tratamiento ajustado al riesgo de recurrencia ${ }^{16}$, con resultados buenos en cuanto a recidivas y supervivencia, evitando en la mayoría de los casos cirugías complejas con linfadenectomías, tratamiento ablativo con $\mathrm{I}^{131}$ y terapia de supresión de TSH.

En cuanto a las persistencias, todos los pacientes cumplieron los criterios de curación a los 6 meses, y por lo tanto no tuvieron persistencias de la enfermedad tras el tratamiento quirúrgico inicial. Hay que tener en cuenta, al igual que en otros estudios ${ }^{17}$, que los pacientes sometidos inicialmente a lobectomía en quienes se completó la tiroidectomía en los primeros 6 meses y se evidenció un MCPT en el lóbulo contralateral no han sido considerados como pacientes con persistencia local, sino que se ha considerado como parte de la cirugía inicial. Al igual que en este trabajo, las persistencias descritas en la literatura científica son bajas, como en el trabajo de Kim, et al. ${ }^{18}$, en el cual se describe una tasa de persistencias del $0.5 \%$.

Respecto a las recurrencias del MCPT, hay que tener en cuenta dos aspectos. Por un lado, el tiempo de seguimiento, ya que pueden tardar tiempo en recidivar, tal como ocurrió en nuestro caso. Por otro lado, los criterios para definir una recidiva no son homogéneos de unas series a otras. Esto justifica la oscilación tan grande en los porcentajes de recidivas documentados en la literatura, que van desde un
$0.4 \%$ hasta un $19 \%^{1,12,17-34}$. En la serie presentada fue del $0.6 \%$, correspondiendo a un solo paciente del grupo de alto riesgo de recurrencia, cuya recidiva tuvo lugar a los 8 años de seguimiento. Esta baja cifra de recidivas posiblemente se deba al alto número de casos incidentales (que suelen tener mejor pronóstico $)^{1,21}$, al elevado porcentaje de pacientes con muy bajo o bajo riesgo de recurrencia, y al tratamiento más agresivo de los pacientes con alto riesgo de recurrencia, en los que se realizaron más linfadenectomías cervicales y se administró $I^{131}$ con mayor frecuencia.

En este estudio, debido a la presencia de un único caso de recidiva, no se han evidenciado factores asociados a la recidiva de la enfermedad. Posiblemente, el tratamiento más agresivo en función de los grupos de riesgo ha influido en este hecho. Entre los factores de riesgo independientes para el desarrollo de recurrencias del MCPT descritos en la literatura científica destacan el diagnóstico clínico, el sexo masculino, la edad menor de 45 años, el tamaño > 5-8 mm según las series, la suma de diámetros de los focos tumorales $>1 \mathrm{~cm}$, la ausencia de cápsula tumoral, la multifocalidad, la bilateralidad, la invasión capsular, la invasión extracapsular, la presencia de adenopatías metastásicas, el número de adenopatías metastásicas $\geq 4$, la ratio de adenopatías metastásicas (ganglios metastásicos/total de ganglios aislados) $>0.44$, la ausencia de enfermedades tiroideas autoinmunitarias (ausencia de enfermedad de Graves y de tiroiditis de Hashimoto), el patrón histológico sólido, la elevación de la tiroglobulina estimulada, la ausencia de terapia metabólica con $1^{131}$ y la mayor duración del seguimiento ${ }^{1,12,17-34}$.

En cuanto a la mortalidad asociada con el MCPT, se ha documentado una tasa del $0-2.2 \%$, según las series $^{17,24,27,31,33}$. De manera similar, en este trabajo ningún paciente falleció debido a la enfermedad durante el seguimiento, lo que pone de manifiesto el buen pronóstico del MCPT a largo plazo cuando se realiza un tratamiento adecuado.

\section{Conclusiones}

El MCPT tratado en función de su riesgo de recurrencia presenta un buen pronóstico a largo plazo, sin persistencias, con un bajo porcentaje de recurrencias y ausencia de mortalidad asociada a la enfermedad.

\section{Conflicto de intereses}

Los autores declaran que no existe conflicto de intereses. 


\section{Responsabilidades éticas}

Protección de personas y animales. Los autores declaran que para esta investigación no se han realizado experimentos en seres humanos ni en animales.

Confidencialidad de los datos. Los autores declaran que han seguido los protocolos de su centro de trabajo sobre la publicación de datos de pacientes.

Derecho a la privacidad y al consentimiento in-

formado. Los autores han obtenido el consentimiento informado de los pacientes y/o sujetos referidos en el artículo. Este documento obra en poder del autor de correspondencia.

\section{Bibliografía}

1. Roti E, degli Uberti EC, Bondanelli M, Braverman LE. Thyroid papillary microcarcinoma: a descriptive and meta-analysis study. Eur J Endocrinol. 2008:159:659-73.

2. Soares P, Celestino R, Gaspar da Rocha A, Sobrinho-Simões M. Papillary thyroid microcarcinoma: how to diagnose and manage this epidemic? Int J Surg Pathol. 2014;22:113-9.

3. Ríos A, Rodríguez JM, Galindo PJ, Montoya M, Tebar FJ, Sola J, et al. Utility of fine-needle aspiration for diagnosis carcinoma associated with multinodular goitre. Clin Endocrinol (Oxf). 2004;61:732-7.

4. Ríos A, Torregrosa B, Rodríguez JM, Rodríguez D, Cepero A, Abellán MD, et al. Ultrasonographic risk factors of malignancy in the thyroid nodule. Importance of ultrasound Doppler. A prospective study. Langenbecks Arch Surg. 2016;401:839-49.

5. Ríos A, Rodríguez JM, Navas D, Cepero A, Torregrosa NM, Balsalobre MD, et al. Family screening in familial papillary carcinoma: the early detection of thyroid disease. Ann Surg Oncol. 2016;23:2564-70

6. Ríos A, Rodríguez JM, Balsalobre MD, Tebar FJ, Parrilla P. The value of various definitions of intrathoracic goiter for predicting intra-operative and postoperative complications. Surgery. 2010;147:233-8.

7. Carlini M, Giovannini C, Castaldi F, Mercadante E, Dell'Avanzato R, Zazza S, et al. High risk for microcarcinoma in thyroid benign diseases. Incidence in a one year period of total thyroidectomies. J Exp Clin Cancer Res. 2005;24:231-6.

8. Sakorafas GH, Stafyla V, Kolettis T, Tolumis G, Kassaras G, Peros G. Microscopic papillary thyroid cancer as an incidental finding in patients treated surgically for presumably benign thyroid disease. J Postgrad Med. 2007:53:23-6.

9. Slijepcevic N, Zivaljevic V, Marinkovic J, Sipetic S, Diklic A, Paunovic I. Retrospective evaluation of the incidental finding of 403 papillary thyroid microcarcinomas in 2466 patients undergoing thyroid surgery for presumed benign thyroid disease. BMC Cancer. 2015;15:330.

10. Miyauchi A. Clinical trials of active surveillance of papillary microcarcinoma of the thyroid. World J Surg. 2016;40:516-22.

11. Gershinsky M, Barnett-Griness O, Stein N, Hirsch D, Tzvetov G, Bardicef $O$, et al. Total versus hemithyroidectomy for microscopic papillary thyroid cancer. J Endocrinol Invest. 2012;35:464-8.

12. Xue S, Wang P, Liu J, Chen G. Total thyroidectomy may be more reasonable as initial surgery in unilateral multifocal papillary thyroid microcarcinoma: a single-center experience. World J Surg Oncol. 2017;15:62.

13. Besic N, Zgajnar J, Hocevar M, Petric R. Extent of thyroidectomy and lymphadenectomy in 254 patients with papillary thyroid microcarcinoma: a single-institution experience. Ann Surg Oncol. 2009;16:920-8.

14. Chang YW, Kim HS, Kim HY, Lee JB, Bae JW, Son GS. Should central lymph node dissection be considered for all papillary thyroid microcarcinoma? Asian J Surg. 2016;39:197-201.
15. Cecoli F, Ceresola EM, Altrinetti V, Cabria M, Cappagli M, Montepagani A, et al. Therapeutic strategies and clinical outcome in papillary thyroid microcarcinoma: a multicenter observational study. Eur Thyroid J. 2016;5:180-6.

16. Pitoia F, Ward L, Wohllk N, Friguglietti C, Tomimori E, Gauna A, et al. Recommendations of the Latin American Thyroid Society on diagnosis and management of differentiated thyroid cancer. Arq Bras Endocrinol Metabol. 2009:53:884-7.

17. Chow SM, Law SC, Chan JK, Au SK, Yau S, Lau WH. Papillary microcarcinoma of the thyroid - prognostic significance of lymph node metastasis and multifocality. Cancer. 2003;98:31-40.

18. Kim KJ, Kim SM, Lee YS, Chung WY, Chang HS, Park CS. Prognostic significance of tumor multifocality in papillary thyroid carcinoma and its relationship with primary tumor size: a retrospective study of 2,309 consecutive patients. Ann Surg Oncol. 2015;22:125-31.

19. Jeon MJ, Kim WG, Kwon H, Kim M, Park S, Oh HS, et al. Clinical outcomes after delayed thyroid surgery in patients with papillary thyroid microcarcinoma. Eur J Endocrinol. 2017;177:25-31.

20. Choi SY, Cho JK, Moon JH, Son YI. Metastatic lymph node ratio of central neck compartment has predictive values for locoregional recurrence in papillary thyroid microcarcinoma. Clin Exp Otorhinolaryngol. 2016;9:75-9.

21. Gschwandtner E, Klatte T, Swietek N, Bures C, Kober F, Ott J, et al. Increase of papillary thyroid microcarcinoma and a plea for restrictive treatment: a retrospective study of 1,391 prospective documented patients. Surgery. 2016;159:503-11.

22. Lee J, Song Y, Soh EY. Central lymph node metastasis is an important prognostic factor in patients with papillary thyroid microcarcinoma. J Korean Med Sci. 2014;29:48-52.

23. Ardito G, Revelli L, Giustozzi E, Salvatori M, Fadda G, Ardito F, et al. Aggressive papillary thyroid microcarcinoma: prognostic factors and therapeutic strategy. Clin Nucl Med. 2013;38:25-8.

24. Kim WY, Kim HY, Son GS, Bae JW, Lee JB. Clinicopathological, immunohistochemical factors and recurrence associated with extrathyroidal extension in papillary thyroid microcarcinoma. J Cancer Res Ther. 2014:10:50-5.

25. Riss JC, Peyrottes I, Chamorey E, Haudebourg J, Sudaka A, Benisvy D, et al. Prognostic impact of tumour multifocality in thyroid papillary microcarcinoma based on a series of 160 cases. Eur Ann Otorhinolaryngol Head Neck Dis. 2012;129:175-8.

26. Mantinan B, Rego-Iraeta A, Larrañaga A, Fluiters E, Sánchez-Sobrino $P$, Garcia-Mayor RV. Factors influencing the outcome of patients with incidental papillary thyroid microcarcinoma. J Thyroid Res. 2012;2012: 469397.

27. Buffet C, Golmard JL, Hoang C, Trésallet C, Du Pasquier Fédiaevsky L, Fierrard $\mathrm{H}$, et al. Scoring system for predicting recurrences in patients with papillary thyroid microcarcinoma. Eur J Endocrinol. 2012;167: 267-75.

28. Cho JK, Kim JY, Jeong CY, Jung EJ, Park ST, Jeong SH, et al. Clinical features and prognostic factors in papillary thyroid microcarcinoma depends on age. J Korean Surg Soc. 2012;82:281-7.

29. Lombardi CP, Bellantone R, De Crea C, Paladino NC, Fadda G, Salvatori M, et al. Papillary thyroid microcarcinoma: extrathyroidal extension, lymph node metastases, and risk factors for recurrence in a high prevalence of goiter area. World J Surg. 2010;34:1214-21.

30. Giordano D, Gradoni P, Oretti G, Molina E, Ferri T. Treatment and prognostic factors of papillary thyroid microcarcinoma. Clin Otolaryngol. 2010;35:118-24.

31. Mercante G, Frasoldati A, Pedroni C, Formisano D, Renna L, Piana S, et al. Prognostic factors affecting neck lymph node recurrence and distant metastasis in papillary microcarcinoma of the thyroid: results of a study in 445 patients. Thyroid. 2009;19:707-16.

32. Hay ID, Hutchinson ME, Gonzalez-Losada T, Mclver B, Reinalda ME, Grant CS, et al. Papillary thyroid microcarcinoma: a study of 900 cases observed in a 60-year period. Surgery. 2008;144:980-7; discussion 987-8.

33. Noguchi S, Yamashita H, Uchino S, Watanabe S. Papillary microcarcinoma. World J Surg. 2008:32:747-53.

34. Roti E, Rossi R, Trasforini G, Bertelli F, Ambrosio MR, Busutti L, et al. Clinical and histological characteristics of papillary thyroid microcarcinoma: results of a retrospective study in 243 patients. J Clin Endocrinol Metab. 2006;91:2171-8. 